2006, 110, 14529-14530

Published on Web 07/13/2006

\title{
Diffusion in Confinement: Agreement between Experiments Better than Expected
}

\author{
E. Beerdsen*,† and B. Smit ${ }^{\dagger, \hbar}$ \\ Van't Hoff Institute for Molecular Sciences (HIMS), University of Amsterdam, Nieuwe Achtergracht 166, \\ 1018 WV Amsterdam, The Netherlands, and Centre Européen de Calcul Atomique et Moléculaire (CECAM), \\ Ecole Normale Supérieure, 46, Allée d'Italie, 69007 Lyon, France
}

Received: May 10, 2006; In Final Form: June 16, 2006

The diffusion of alkanes in nanoporous materials as measured by different experimental techniques is thought to be highly dependent on the measuring technique employed. However, when the data are corrected for the loading at which the measurement was performed, the different data series correspond with each other much better than expected.

\section{Introduction}

Detailed knowledge of the diffusion behavior of hydrocarbons in molecular sieves is of importance in many petrochemical processes. Zeolites are nanoporous materials, often used as model structures to study diffusion in confinement, owing to their regular, well-defined crystalline nature. Although there are over 130 different known zeolite topologies, most groups focus on the understanding of the diffusion in silicalite (MFI) because this material is very well characterized and the MFI structure is used in many industrial applications. ${ }^{1-12}$

Talu et al. ${ }^{1}$ made a comparison of the various techniques used to measure diffusion in confinement. Figure 1 is based on their results. This figure illustrates why, until recently, it was considered common knowledge in zeolite science that diffusion measurements obtained by different techniques rarely correspond to one another and that the differences between different experimental techniques and simulations can be as great as 5 orders of magnitude. However, a closer inspection of the experimental data shows that the compilation in Figure 1 has been made with the assumption (the so-called Darken approximation) that the diffusion coefficient is independent of the loading. ${ }^{13}$ For a long time this was considered a very reasonable assumption in MFI, ${ }^{14}$ and, in accordance with this view, no corrections have been made for loading effects in plotting the data. However, in recent years, molecular simulations have shown that for MFI, as for other zeolites, there is a significant loading dependence. ${ }^{15}$ This observation motivated us to reanalyze the experimental data.

A second motivation lies in the desirability of a comparison between different data sets. Most force fields have been optimized and validated using adsorption data. It is therefore very important to know whether these force fields also give an accurate description of the diffusion behavior. Until now, this question could not be addressed because the large differences between the various data made it impossible to draw any conclusions.

\footnotetext{
* Corresponding author. E-mail: beerdsen@science.uva.nl.

¿ University of Amsterdam.

$\doteqdot$ Ecole Normale Supérieure.
}

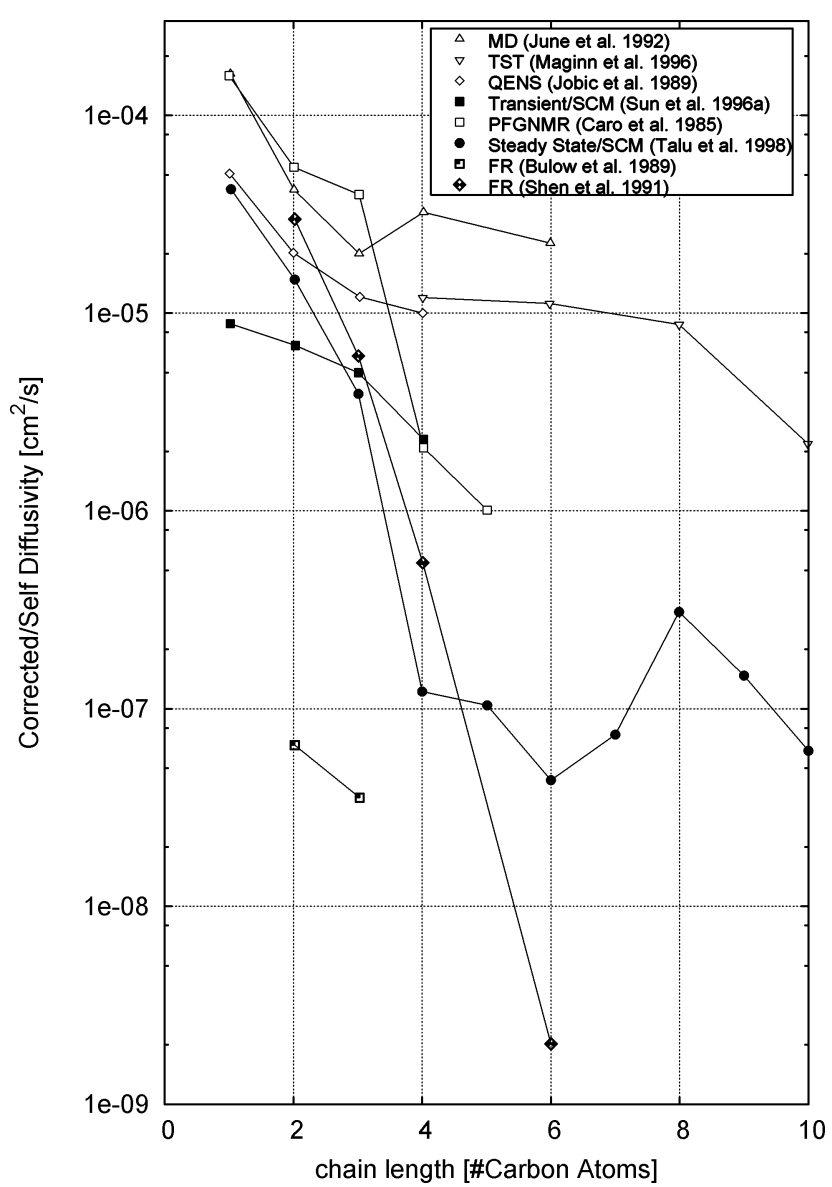

Figure 1. Alkane diffusion in MFI as a function of chain length, based on a graph from Talu et al., ${ }^{1}$ summarizing data from several groups obtained by various methods, at $300 \mathrm{~K}$.

In this letter, we strive to solve the riddle behind the graph: Why is the measured diffusion so high in some methods, while it comes out so low in others? We pick out a simple test case: the diffusion of methane, which differs more than 1 order of magnitude between the lowest and the highest measurement. 


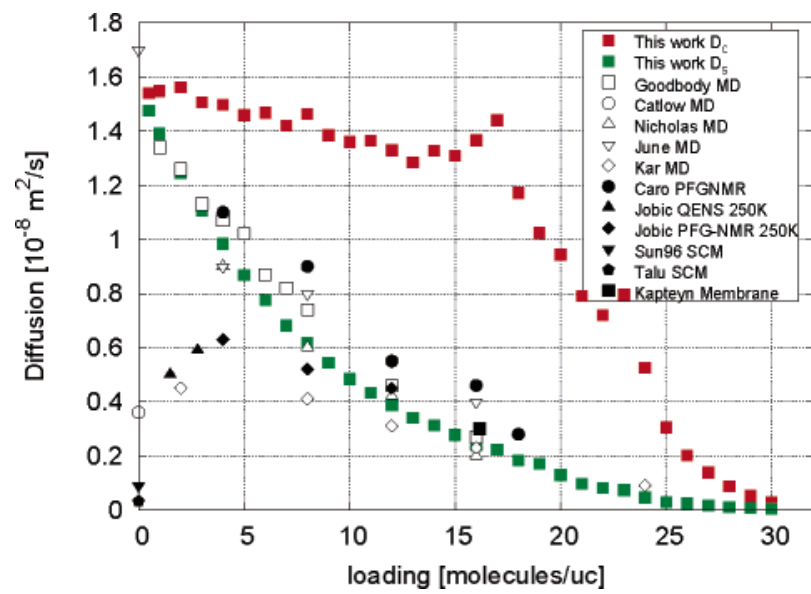

Figure 2. Recent simulation results for the diffusion of methane in MFI-type silica as a function of the adsorbate loading, ${ }^{15}$ together with results obtained by several other groups through simulation ${ }^{2-6}$ and experiment. ${ }^{1,7-10}$ Data were obtained at $300 \mathrm{~K}$, except for the results of Jobic et al., which were obtained at $250 \mathrm{~K}$.

\section{Measuring Diffusion}

Diffusion can be expressed in a diffusion coefficient in several ways. In practical experiments, such as measurements of the uptake and permeation rate, the diffusion measured is usually the transport diffusion coefficient $D_{\mathrm{t}}$, defined by Fick's law. ${ }^{14,16}$ To obtain a quantity that is independent of concentration gradients, $D_{\mathrm{t}}$ can be converted to the corrected diffusivity $D_{\mathrm{c}},{ }^{17,18}$ also known as the Maxwell-Stefan, Darken, or collective diffusion coefficient. It is the collective diffusion behavior of all adsorbate particles, and it can be interpreted as the movement of the center of mass of all particles together. Another common measure of diffusivity is the self-diffusion coefficient $D_{\mathrm{s}}$. It is the diffusion of a single tagged particle moving around in a sea of other particles. This is the diffusion coefficient that can be obtained by microscopic methods, such as pulsed field gradient NMR (PFG-NMR). In general, the corrected diffusion is higher than the self-diffusion because the corrected diffusion contains interparticle correlations, which have a positive contribution, or, viewed differently, the self-diffusion is lowered by singleparticle back-correlations (the increased probability of a particle jumping back to its previous position because this position has a higher probability of being empty).

\section{Results}

Figure 2 shows our recent simulation results for the diffusion of methane in MFI-type silica as a function of the adsorbate loading, ${ }^{15}$ together with results obtained by several other groups through simulation ${ }^{2-6}$ and experiment. ${ }^{1,7-10}$ Note that the potential parameters used in the simulations have been fitted to adsorption data, not diffusion data. ${ }^{19}$ The experimental data (black symbols) are self-diffusion coefficients, except those obtained by single-crystal membrane (SCM) measurements, which should be corrected diffusivities. The results have been plotted against the loading, as reported in the original papers, wherever possible. Unspecified loadings have been estimated from the reported pressures with the aid of a calculated adsorption isotherm.

Although some results show a marked deviation-both SCM studies, ${ }^{1,9}$ carried out at the zero-loading limit, yielded a diffusion that is much slower than that found by other methodsthe overall correlation between different experimental and simulation results is remarkably good, much better than we expected from methods that are often said to be irreconcilable.

Furthermore, we conclude that the one-order difference in methane diffusion found in Figure 1 can be ascribed to the differences in loading at which the different experiments have been carried out. Larger differences are to be expected for longer alkanes.

\section{Concluding Remarks}

To compare diffusion measurements obtained by different methods, it is very important to know the loadings at which the measurements were performed. Loadings, if known, or pressures should be mentioned along with the reported diffusivity data. In addition, we can conclude that the methane force field of ref 19 predicts the diffusion coefficient accurately.

The deviation observed between the single-crystal studies and most other studies is probably due to the existence of both internal and external diffusional barriers. The more macroscopic a measurement method, the larger the influence of the internal barriers. ${ }^{12}$ As the internal barriers are relatively small for methane in MFI, it is expected that the diffusivities obtained by macroscopic and microscopic methods will be further apart for longer alkanes.

Acknowledgment. This work is supported by the EC through the Marie Curie EXT Project MEXT-CT-2005-023311, The Netherlands Research Council (CW), and the Deutsche Forschungsgemeinschaft (DFG, priority program SPP 1155). We thank NWO/NCF for computational resources.

\section{References and Notes}

(1) Talu, O.; Sun, M. S.; Shah, D. B. AIChE J. 1998, 44, 681.

(2) Goodbody, S. J.; Watanabe, J. K.; Gowan, D. M.; Walton, J. P. R. B.; Quirke, N. J. Chem. Soc., Faraday Trans. 1991, 87, 1951.

(3) Catlow, C. R. A.; Freeman, C. M.; Vessal, B.; Tomlinson, S. M.; Leslie, M. J. Chem. Soc., Faraday Trans. 1991, 87, 1947.

(4) Nicholas, J. B.; Trouw, F. R.; Mertz, J. E.; Iton, L. E.; Hopfinger, A. J. J. Phys. Chem. 1993, 97, 4149.

(5) Kar, S.; Chakravarty, C. J. Phys. Chem. A 2001, 105, 5785.

(6) June, R. L.; Bell, A. T.; Theodorou, D. N. J. Phys. Chem. 1990, 94, 8232.

(7) Caro, J.; Bulow, M.; Schirmer, W.; Karger, J.; Heink, W.; Pfeifer, H. J. Chem. Soc., Faraday Trans. 1985, 81, 2541.

(8) Jobic, H.; Bee, M.; Caro, J.; Bulow, M.; Karger, J. J. Chem. Soc., Faraday Trans. 1989, 85, 4201.

(9) Sun, M. S.; Talu, O.; Shah, D. B. AIChE J. 1996, 42, 3001.

(10) Kapteyn, F.; Bakker, W. J. W.; Zheng, G.; Moulijn, J. A. Chem. Eng. J. 1995, 57, 145-153.

(11) Vasenkov, S.; Böhlmann, W.; Galvosas, P.; Geier, O.; Liu, H.; Kärger, J. J. Phys. Chem. B 2001, 105, 5922.

(12) Vasenkov, S.; Kärger, J. Microporous Mesoporous Mater. 2002. 55,139 .

(13) Auerbach, S. M. Int. Rev. Phys. Chem. 2000, 19, 155.

(14) Kaerger, J.; Ruthven, D. M. Diffusion in Zeolites and Other Microporous Solids; John Wiley \& Sons: New York, 1992.

(15) Beerdsen, E.; Dubbeldam, D.; Smit, B. Phys. Rev. Lett. 2005, 95 , 164505.

(16) Krishna, R.; Paschek, D. Chem. Phys. Lett. 2001, 33, 278.

(17) Ramanan, H.; Auerbach, S. M. In NATO-ASI Series C: Fluid Transport in Nanopores; Fraissard, J., Conner, W. C., Eds.; Kluwer Academic Publishers: Dordrecht, The Netherlands, 2004.

(18) Krishna, R.; Paschek, D.; Baur, R. Microporous Mesoporous Mater. 2004, 76, 233-246.

(19) Dubbeldam, D.; Calero, S.; Vlugt, T. J. H.; Krishna, R.; Maesen, T. L. M.; Beerdsen, E.; Smit, B. Phys. Rev. Lett. 2004, 93, 088302-1. 